\title{
Psychiatrischer Notfalldienst Stadt Zürich, 11./12. Juni 2002
}

\section{Romann}

Der Psychiatrische Notfalldienst in der Stadt Zürich beginnt um 12 Uhr mittags und dauert 24 Stunden. Kurz vor 12 Uhr beende ich meine letzte reguläre Therapiestunde und melde mich beim Ärztefon. Die dort diensttuenden Krankenschwestern und Pfleger ersparen uns mit ihrer sorgfältigen Triage manch unnötigen Gang, sie sind in der Regel freundlich und ausgesprochen kompetent und bringen es fertig, am Telefon sehr viel Ruhe auszustrahlen. Nur einmal hat mich die zuständige Person etwas aus der Fassung gebracht, indem sie mich nach einer reichlich kurzen Nacht morgens um sieben Uhr nach meiner Natelnummer gefragt hat, die natürlich schon seit bald 20 Stunden im Ärztefon bekannt war ...

Kurz vor 13 Uhr kommt der erste Anruf: ein Psychiater aus einem Landbezirk möchte, dass ich eine Patientin, die bei ihm Anfang nächster Woche einen ersten Termin hat, anschaue. Sie ist offensichtlich psychotisch und es ist unklar, ob ihr Zustand ein Zuwarten bis nächste Woche erlaubt. Ein Gespräch mit dem Kollegen, den ich persönlich kenne und sehr schätze, bringt noch etwas Klärung: die noch junge Patientin wurde ihm von deren Eltern angemeldet, die im gleichen Dorf leben, in dem er praktiziert. Deshalb die etwas komplizierte Situation, dass die Patientin aus Zürich zu ihm aufs Land gehen wird. Die Eltern, die sich sehr um die Tochter sorgen, bemühen sich, dass die Tochter, die sich selber wahrscheinlich gar nicht als krank erlebt, kompetente Hilfe erhält.

Ich rufe die junge Frau an, es ist eine Studentin, knapp dreissig Jahre alt. Sie ist bereit, mit mir zu reden, möchte aber nicht zu mir in die Praxis kommen, dazu fühlt sie sich nicht in der Lage. Ihr Freund sei bei ihr.

Um 13.20 Uhr komme ich in der etwas heruntergekommenen Studentenwohngemeinschaft an. Haben wir früher auch so karg möbliert gelebt, mit so minimalem Komfort? Wahrscheinlich schon, ich bin wohl etwas wohlstandsverwöhnt mittlerweile. Die Patientin liegt auf ihrem Bett, trotz ziemlicher Hitze in Kissen und Decke eingewickelt, sie wirkt sehr jung, zerbrechlich, sehr verängstigt und angespannt. Der Freund, ein schlanker ebenfalls angespannt wirkender Mann, hat mir die Türe geöffnet, auf ihre
Bitte hin bleibt er im Raum anwesend. Mir ist das recht, so habe ich eine Quelle für die hier unerlässliche Fremdanamnese in Anwesenheit der Patientin selber, deren psychotische Angst ich durch grösstmögliche Transparenz zu lindern versuche. Sie schaut mich genau an, versucht zu ergründen, ob sie mir trauen kann. Ich bitte sie, mir zu erzählen, was sie so verängstigt. Nach ein paar Minuten hat sie soweit Vertrauen gefasst, dass sie mir schildern kann, was ihrer Meinung nach mit ihr passiert ist. Sie war alleine in den Ferien und geriet in die Hände einer sektenartigen Organisation. Sie schildert sehr anschaulich, an welchen Zeichen sie erkannt hat, dass mit ihr ein grauenhaftes Experiment gemacht werde. Sie sei sich über die Zielsetzung des Experimentes nicht im klaren, zuerst aber einmal müsse sie wissen, ob das die Wahrheit sei. Sie schaut mich eindringlich an: «Sie müssen mir sagen, ob ich in einem Experiment bin oder nicht!» «Was wäre, wenn es kein Experiment ist?», frage ich zurück. Das wäre noch viel schlimmer, dann wäre es ernst, meint sie mit leiser Stimme und blickt mich hilfesuchend an. Ich bin sehr berührt von ihrer verzweifelten Suche nach Sinn und Bedeutung dessen, was sie subjektiv erlebt hat. Sie ist deutlich wahnhaft, aber im Gespräch gut erreichbar. Sie hat funktionierende soziale Beziehungen und Eltern, die sich um sie kümmern, ist bei einem guten Psychiater angemeldet, ihre Chancen stehen nicht schlecht. Ich entschliesse mich, ihre Frage in etwa so zu beantworten: «Ich glaube nicht, dass Sie in einem Experiment sind. Ich weiss nicht, was mit Ihnen passiert ist, aber ich bin sicher, dass Sie nicht unmittelbar bedroht sind. Sie haben Zeit und können sich in den nächsten Wochen mit Dr. X gründlich damit befassen, welche Bedeutung das alles für Ihr Leben hat.» Sie wird erstaunlich ruhig, hört genau zu, entspannt sich sichtlich. Ich glaube, sie kann bis zu ihrem Termin zuwarten. Natürlich wäre es hilfreich, wenn sie Medikamente akzeptieren würde, aber wie so viele in ihrer Situation lehnt sie das kategorisch ab. Ich lasse trotzdem wenigstens ein paar Temesta da, im Zweifelsfall für den Freund, der mittlerweile sehr mitgenommen wirkt. Er bestätigt mir die fassbaren Daten, kann mir auch versichern, dass genügend Leute da sind, damit die Patientin in den nächsten Tagen 
nie alleine sein muss. Mit ihr selber verabrede ich, dass sie mich am nächsten Morgen anruft, das wird mir einen weiteren Hinweis auf die «Vertragsfähigkeit» der Patientin geben.

Nach 50 Minuten kann ich die Wohnung verlassen, draussen ist heller Tag in Zürichs Langstrasse-Quartier - irgendwie unwirklich nach der intensiven Begegnung in der abgedunkelten Wohnung.

Ich fahre mit dem Auto in die Praxis zurück, fühle mich etwas verloren und leer. Ich versuche, meine Gedanken zu ordnen: so aus der Welt gefallen ist die junge Frau, das spüre ich nachhaltig.

Tatsächlich ruft sie am nächsten Morgen zur vereinbarten Zeit an, die Stimme klingt zuversichtlicher, ich hoffe, sie findet den Weg zu einer Psychotherapie und zu einem hilfreichen Gebrauch von Medikamenten.

Zurück in der Praxis habe ich zunächst Ruhe, Zeit, Administratives zu erledigen, Zeugnisse warten schon seit längerem usw. Um 16 Uhr geht es wieder los.

Eine Sozialarbeiterin ruft an, sie hat eine suizidale Klientin bei sich. Der Frau wurde soeben eröffnet, dass man ihr das Sorgerecht für ihren dreijährigen Sohn entzieht, «bei einem Münchhausen-by-proxi-Syndrom». Die Stimme der Sozialarbeiterin tönt fragend: weiss die Psychiaterin wohl, was das ist. Sie weiss es und es gehört zu den wenigen Dingen, bei denen meine Empathie versagt: artifizielle Störung (absichtliches Erzeugen oder Vortäuschen von körperlichen oder psychischen Störungen oder Behinderungen), so beschreibt das ICH-10-Manual das Münchhausen-Syndrom, by proxi bedeutet, dass die Mutter mit ihrem Kind so verfährt.

Das wird nun wieder eine derjenigen Patienten/-innen sein, die mein Kommen keinesfalls begrüssen wird. Therapeutisch werde ich ihr wenig bis nichts zu bieten haben, es wird auf einen fürsorgerischen Freiheitsentzug hinauslaufen. Der Gesetzgeber hat das eigentlich als eine behördliche Aufgabe konzipiert, im Kanton Zürich sind es aber wir niedergelassenen Ärztinnen und Ärzte, in der Stadt vorab die Psychiater/innen, die diese meistens sehr undankbare Aufgabe zu übernehmen haben.

Ich finde im Büro des Sozialzentrums eine korpulente Serbin, Ende Vierzig, sie hat den Sohn spät bekommen. Sie selber lebt schon seit Jahren in der Schweiz und spricht gut deutsch. Der Vater des Kindes ist als serbischer Offizier im Kosovo von einem Heckenschützen erschossen worden. Die Frau redet ununterbrochen und eindringlich, versucht mir zu erklären, dass sie immer nur das beste für ihren Sohn gewollt habe.
Sie spricht pausenlos, die Stimmung ist äusserst angespannt. Sie kann nicht verstehen, was man ihr vorwirft, wehrt sich mit allen Mitteln und ist mit einer Hospitalisation natürlich nicht einverstanden. Sie bringt ihre Mutter ins Spiel, die bei ihr zu Hause wohne und niemanden habe, wenn sie nicht nach Hause komme. Um das wird sich später die Sozialarbeiterin kümmern müssen, sie macht jetzt schon Überstunden und es wird noch ein Weilchen dauern. Ich schaue die Patientin an: weiss sie wirklich nicht, dass sie ihr Kind misshandelt hat, lebensgefährlich, wie ich nachher von der Sozialarbeiterin erfahre. Es geht hin und her, ich rufe mittlerweile in der Klinik an, um die Patientin anzumelden. Ich erhalte den absolut letzten Platz - mein Dienst dauert noch bis zum nächsten Mittag! Die Bettensituation ist wieder einmal katastrophal und da schicke ich nun eine Patientin, die von einem Klinikaufenthalt kaum profitieren wird. Ihr Gesicht ist ausdruckslos, ich kann sie innerlich nicht erreichen. Welche Scheusslichkeiten hat diese Frau wohl selber erdulden müssen? Und welche hat ihr Mann begangen, im Kosovo, aber vielleicht auch mit ihr? Sie tut mir sehr leid, gleichzeitig empfinde ich aber auch eine starke Ablehnung. Wie wird der kleine Junge wohl mit dieser Hypothek aufwachsen? Meine Ambivalenz verleitet mich wohl zum nachfolgenden Entscheid, der sich sehr schnell als kapitaler Fehler erweisen sollte: ich möchte ihr die Ambulanz ersparen, die Klinik ist keine 10 Minuten Autofahrt von hier entfernt, ich frage die Sozialarbeiterin, ob sie bereit ist, mit mir zusammen die Patientin in meinem Auto in die Klinik zu fahren. Wir kommen nicht weit: kaum auf der Strasse schlägt die Patientin mir den Schlüsselbund aus der Hand und rennt weg. Obwohl ich zuerst inmitten der auf das Grünlicht zufahrenden Autos meine Schlüssel zusammensuchen muss, bin ich einiges schneller als sie, renne hinter ihr her und packe sie nun ziemlich energisch an, die Sozialarbeiterin nimmt den anderen Arm, gemeinsam führen wir die Frau zurück ins Büro und ich fordere die Ambulanz an. Während wir warten unendlich lange, wie mir scheinen will -, bin ich angespannt wie eine zu stark aufgezogene Feder, jederzeit bereit, auf einen allfälligen Gewaltausbruch zu reagieren.

Obwohl ich den Tagesarzt gebeten habe, mich über den weiteren Verlauf zu orientieren, höre ich nichts mehr von der Frau. Seit dem Inkrafttreten des neuen Patientenrechtes erhalten wir nicht mehr automatisch einen Austrittsbericht, sondern nur noch auf Anfrage und nur, wenn die Patientin ihr Einverständnis gibt, eine sehr unbefriedigende Situation, schliesslich ge- 
hören diese Rückmeldungen zu unserer Fortbildung.

Der nächste Anruf erreicht mich, bevor ich wieder in der Praxis bin, und verheisst wieder nichts Gutes: eine 16jährige Asylsuchende will sich umbringen, weil ihr Freund sie verlassen hat. Angerufen hat die für das Mädchen zuständige Betreuerin der Asylorganisation.

Wieder finde ich eine sehr karge Wohnung vor, noch um einiges spärlicher möbliert. Sind wir da wirklich in Zürich? Drei 16jährige Asylsuchende leben hier zusammen, betreut von einer knapp 20jährigen sogenannten Mediatorin und dem Fachdienst für unbegleitete Minderjährige. Die Betreuerin ist gereizt und erschöpft, sie möchte, dass sofort gehandelt wird. Ich muss mir halt trotzdem zuerst ein eigenes Bild machen, schliesslich geht es um einen fürsorgerischen Freiheitsentzug bei einer Minderjährigen! Nach kurzer Zeit verstehe ich die Gereiztheit der Betreuerin allerdings bestens: das Mädchen schreit mich an: «Du musst meinen Freund anrufen, sonst bringe ich mich um». Der Freund, so stellt sich heraus, ist ein sechs Jahre älterer Albaner, sie selber eine Roma aus dem Kosovo, seit zwei Jahren hier, ohne Familie oder sonstige Verwandte, in einer ganz anderen Kultur, ohne Ausbildung, mit ungenügenden Sprachkenntnissen: was hat sie überhaupt für Perspektiven? Und was mache ich jetzt mir ihr? Das Kriseninterventionszentrum kommt nicht in Frage, dort war sie schon vor etwa einem halben Jahr. Der damalige Besuch endete mit einem Suizidversuch mit Tabletten! Es ist wirklich gefährlich, auch wenn die ganze Szene jetzt sehr demonstrativ wirkt. Sie schreit und heult, bedeckt das Gesicht mit den Händen, schaut mich aber zwischen den Fingern kurz prüfend an: wirkt das Ganze auch genügend auf mich? Ich bin - im Gegensatz zur Betreuerin - durchaus bereit, mit dem Mann zu reden, auch weil ich ihm die Konsequenzen seines ziemlich verantwortungslosen Umgangs mit der jungen Frau nicht ganz ersparen möchte. Dass sie in ihrem Alter bereits einen Schwangerschaftsabbruch hinter sich hat, empört mich zusätzlich. Ich erreiche ihn an der angegebenen Nummer nicht, seine Schwester ist am Telefon. Zuerst ist sie misstrauisch, dann plötzlich erzählt sie mir sturzflutartig viel mehr, als ich wissen will, möchte mir die Natelnummer des Bruders angeben und ich solle ihn ruhig anrufen, ihm aber auf keinen Fall mitteilen, was sie mir alles gesagt habe. Ich lehne dankend ab. Ich arbeite mit meinem privaten Natel, dessen Nummer ich keinesfalls auf seinem Display deponieren möchte. Nun wird die Patientin ihrerseits wütend: «Warum hast du die Nummer nicht genom- men?» Ich habe genug, erkläre ihr, dass ich sie gegen ihren Willen zu ihrem Schutz in eine Klinik einweisen werde und gehe in die Küche, um alles in die Wege zu leiten und die nötigen Daten von der Betreuerin zu erfragen. Diese ist mittlerweile etwas entspannter, froh, dass sie die Verantwortung nicht mehr tragen muss. Sie ist eine Bosnierin, zu Hause war sie Dozentin für Philosophie, hier muss sie sich um diese Adoleszenten in sehr schwierigen persönlichen und sozialen Situationen kümmern: auch das ist ein Kriegsschicksal! Die Patientin ist noch minderjährig, ich benötige die Hilfe des Kinderpsychiatrischen Dienstes. Die Kinder- und Jugendpsychiatrischen Notfälle sind im ganzen Kanton ein Dauerbrenner: wo können die jungen Menschen untergebracht werden, wenn eine Hospitalisation nötig ist? Die Kollegin im KJPD ist sehr hilfsbereit, sie sucht und findet ein Bett in einer für Jugendliche einigermassen eingerichteten Klinik, wieder warten wir auf die Ambulanz. Die Patientin ist mittlerweile auch in der Küche aufgetaucht, sie hat sich umgestellt, wirkt ganz munter, die obligate Zigarette im Mundwinkel: «Gibst du mir Dein Handy mit» ist die ultimative Forderung an die Betreuerin! Dieser verschlägt es nun doch fast die Sprache, jetzt will sie nur noch nach Hause! Ich bin mittlerweile auch ziemlich müde und denke mir: wieder eine Patientin, der die Psychiatrie nichts zu bieten hat. Was sie bräuchte, das allerdings dringend, ist eine pädagogische Institution, die ihr Struktur und Halt geben könnte. Zudem ist sie fraglos recht intelligent und könnte mit einer Ausbildung einen Weg aus der sozialen Misere finden.

Dem Austrittsbericht, den ich ein paar Tage später erhalte, entnehme ich, dass die Klinik zu ähnlichen Schlussfolgerungen kam. Da es eine entsprechende sozialpädagogische Wohnmöglichkeit nicht gibt, wird die Patientin in die alten unbefriedigenden Verhältnisse entlassen - bis zum nächsten Crash!

Nun fahre ich nach Hause, mein Mann hat mich angerufen, das Essen warte auf mich, es ist mittlerweile schon fast $21.30 \mathrm{Uhr}$. Ich fahre auf unseren Parkplatz, da kommt der nächste Anruf. Die Bahnhofspolizei hat eine Frau auf den Posten genommen, die sich merkwürdig benehme, sie habe versucht, den vorbeifahrenden Autos einen Tritt zu geben und sich dabei eine Schlägerei mit einer Autofahrerin eingehandelt. Ich bekomme die Patientin selber nicht ans Telefon, sie sei in einem anderen Büro, eine Kollegin bemühe sich um sie. Das muss sie nun noch ein bisschen länger tun, erst einmal brauche ich etwas zu essen. Wer schon auf dem Polizeiposten ist, ist in der Regel ganz gut aufgehoben, sage ich mir. 
Nach einer kurzen Mahlzeit fahre ich los und bin kurz nach 22.30 Uhr auf dem Polizeiposten. Wie fast immer sind die Polizistinnen und Polizisten ausgesprochen nett und kooperativ. Der Polizist, der mich angefordert hat, meint, die Frau sei vielleicht psychotisch, sie sei einfach merkwürdig, verhalte sich auffällig. Ich finde eine 24jährige junge Frau vor, die mir einigermassen zusammenhängend erzählen kann, was sich an diesem Abend abgespielt hat. Nur eine leicht verwaschene Sprache deutet darauf hin, dass Alkohol im Spiel sein könnte. Ich frage nach, sie gibt bereitwillig zu, dass Alkohol ein Problem sei, sie deshalb auch in Behandlung sei. Aber heute habe sie nicht viel getrunken! Ich schlage vor, dass wir das einmal überprüfen, sie hat nichts dagegen. Der Atemtest ergibt ein in seiner Deutlichkeit nicht erwartetes Resultat: 2,5 Promille! Wir sind alle überrascht, die Patientin am meisten, dabei habe sie doch fast nichts getrunken! Damit ist einiges klar, unklar ist, was mit ihr geschehen soll diese Nacht. Kann ich sie so nach Hause lassen? Ich hole mir Rat beim Tagesarzt der inneren Medizin im Universitätsspital. Auch dieser Kollege ist sehr hilfsbereit. Seine Meinung: «Wir lassen die Leute auch noch mit 3,5 Promille nach Hause, wenn sie es gewohnt sind, vorzugsweise natürlich nicht alleine.» Wir versuchen noch, jemanden für sie zu mobilisieren, das ist allerdings so kurz vor Mitternacht dann nicht mehr möglich. Also soll sie nach Hause, mit dem Taxi, sie wohnt in der Nähe.

Unter der Türe gibt mir der zuständige Polizist noch seine Visitenkarte: «Melden Sie sich bei mir, wenn die Patientin nicht bezahlt.» Ich bin erfreut und überrascht. Bisher habe ich immer gehört, dass die Polizei nicht zu haben sei für die entstehenden Kosten, solche Patienten/-innen wie die eben gesehene fühlen sich allerdings des öftern auch nicht zuständig für die Rechnung. Dann beginnt ein aufwendiges Prozedere mit Mahnung bis zur Betreibung usw., das viele diensttuende Psychiater nicht auf sich nehmen mögen.

Bis jetzt hat die Patientin noch nicht bezahlt, sollte es dabei bleiben, werde ich auf das freundliche Angebot zurückkommen.

$\mathrm{Zu}$ Hause bin ich etwa um $1 \mathrm{Uhr}$ früh, jetzt bitte keine Anrufe mehr, ich könnte eine Portion Schlaf vertragen.

Nicht nur habe ich einen ungestörten Schlaf, auch am nächsten Morgen bleibt es ruhig. Ich gehe, für alle Fälle ausgerüstet mit Natel und Notizblock, sogar mit meinem Hund ein bisschen in den Wald spazieren, lüfte meinen Kopf aus und bin froh, dass niemand etwas von mir will. 